\title{
Differences between patients with positive and negative views regarding justification of involuntary admission Christina Katsakou*1, Stefan Priebe ${ }^{1}$, Diana Rose ${ }^{1}$, Angela Sweeney ${ }^{2}$ and Ksenija Yeeles ${ }^{1}$
}

Address: ${ }^{1}$ Unit for Social \& Community Psychiatry, Queen Mary University of London, Glen Road, London, E138SP, UK and ${ }^{2}$ Service User Reasearch Enterprise, De Crespigny Park, London, UK

* Corresponding author

\author{
from WPA Thematic Conference. Coercive Treatment in Psychiatry: A Comprehensive Review \\ Dresden, Germany. 6-8 June 2007 \\ Published: 19 December 2007 \\ BMC Psychiatry 2007, 7(SuppI I):S57 doi:10.1 186/I47I-244X-7-SI-S57
}

This abstract is available from: http://www.biomedcentral.com/I47I-244X/7/SI/S57

(c) 2007 Katsakou et al; licensee BioMed Central Ltd.

\section{Background}

Involuntary admission to a psychiatric hospital is often a long, complicated process likely to elicit strong feelings in patients experiencing it. Qualitative research in this area would give us a better understanding of patients' views on this complex experience and help interpret findings from quantitative studies.

\section{Methods}

The present study complements a quantitative investigation on outcomes of involuntary admission. 60 in-depth semi-structured interviews were conducted with patients who had been involuntarily admitted in 4 sites in the England. The sampling and analysis methodology draws on grounded theory. The sample includes participants admitted under different sections of the Mental Health Act. Their experiences of involuntary admission and treatment and their views on the justification of these interventions were explored. Comparisons between participants with overall positive and negative views were performed. Changes in patients' views over time were particularly examined. Interviews were tape-recorded and transcribed verbatim. Data was coded using MAXqda software for qualitative analysis.

\section{Results}

Results indicate that the evaluation of risk and mental illness, as well as perceived violations of autonomy and discrimination shaped participants' views on the justification of their compulsory admission and treatment. Patterns of changing views consisted of gradual realisation of poor mental state and subsequent need for treatment. 